\title{
Image Registration based Cervical Cancer Detection and Segmentation Using ANFIS Classifier
}

\author{
B Karthiga Jaya ${ }^{1 *}$, S Senthil Kumar²
}

\begin{abstract}
Cervical cancer is the leading cancer in women around the world. In this paper, Adaptive Neuro Fuzzy Inference System (ANFIS) classifier based cervical cancer detection and segmentation methodology is proposed. This proposed system consists of the following stages as Image Registration, Feature extraction, Classifications and Segmentation. Fast Fourier Transform (FFT) is used for image registration. Then, Grey Level Co-occurrence Matrix (GLCM), Grey level and trinary features are extracted from the registered cervical image. Next, these extracted features are trained and classified using ANFIS classifier. Morphological operations are now applied over the classified cervical image to detect and segment the cancer region in cervical images. Simulations on large cervical image dataset demonstrate that the proposed cervical cancer detection and segmentation methodology outperforms the state of-the-art methods in terms of sensitivity, specificity and accuracy.
\end{abstract}

Keywords: Cervical cancer- feature extraction- registration- classification- segmentation

Asian Pac J Cancer Prev, 19 (11), 3203-3209

\section{Introduction}

Cervical cancer is the second dangerous disease in women around the world, especially in developing countries. At present, image processing techniques are used to detect and diagnose the cervical cancer in computer aided automatic approach. Human Papilloma virus (HPV) (NCCC, 2010) is the primary reason for cervical cancer in women patients. The internal organs of the cervix of human body are affected by this virus, which leads to the formation of the cancer in cells. The cancer cells in the cervical image are categorized into benign cells and malignant cells. The benign cells are pre-cancerous cells and it is not spread over the inner areas of the cervix region. The malignant cells are the cancerous cells and it is spread over the inner region of the cervix. The cancer in cervix region is identified using Pap smear test or cervigram. In Pap smear test, the cells in the cervix region are tested and its nuclei regions are diagnosed. In cervigram method, the cervical images are used to detect the cancer region in cervix. In present scenario, the cervical cancer can be identified at the final stage which causes sudden death. In many cases, the patients do not feel any irritations or pain in cervix region and it does not trigger any symptoms. The patient can be saved if the cancer is detected at an earlier stage. This is not possible for large population areas or developing countries. It also requires high number of trained physicians or radiologist to detect the cervical cancer in women. Hence, there is a need for automatic detection and diagnosis system for cervical cancer detection. This paper proposes an automatic cervical cancer detection method in cervigrams using classification approach. Figure 1(a) shows the cervigrams of the normal patient and Figure 1(b) shows the cervigrams of the abnormal patients.

In this paper, an automatic system for the detection of cervical tumor is proposed for earlier detection of tumor in female patients. Section 2 presents the conventional methodologies for cervical tumor detection and Section 3 elaborates the proposed cervical tumor detection and segmentation system. Section 4 discusses the experimental results and Section 5 concludes the work.

Literature Survey

Miri et al., (2018) discussed different cervical cancer detection and segmentation methods on the patients from low income groups worldwide. Sachan et al., (2018) depicted and discussed various cervical cancer detection and screening methodologies using Pap smear cell images. The authors discussed impact of different texture features for the detection and segmentation of abnormal regions in cervical regions. Kashyap et al., (2016) proposed cervical cancer detection approach using multi class Support Vector Machine (SVM) on cervical images. The authors applied independent level set approach on cervical images for detecting and segmenting the abnormal regions. Soumya et al., (2016) used contourlet transform on cervical images in order to decompose the cervical image into matrix coefficients. Then, Gabor features were

${ }^{1}$ ECE, Dhanalakshmi Srinivasan Engineering College, ${ }^{2} E E E$, GCE Salem, Tamilnadu, India. *For Correspondence: karthiga.jaya@yahoo.com 
extracted from the decomposed matrix coefficients. These features were classified using SVM classification approach for identifying the abnormal patterns in cervical images.

Dezhao Song et al., (2015) used multimodal entity method to detect the cancer region in cervical images. The authors achieved $83.21 \%$ sensitivity and $94.79 \%$ specificity using entity model on large cervical image dataset. The main limitation of this method was that the outer region of the cancer region was not detected by this method. Chen et al., (2014) used Support Vector Machine (SVM) classifier to detect and classify the cervical cancer in Pap smear cell images. The authors used various kernels in SVM classification approach for improving the classification rate. The classification of this method was based on the texture features and the authors achieved $96.12 \%$ of Pap smear nuclei cell region.

Rama Praba et al., (2013) applied color histogram method on cervical images to remove the irrelevant and inhomogeneous information. The specular reflection of the various regions in cervical image was improved by training the extracted color histogram and texture features using K-Nearest Neighbor (KNN) classifier. The authors achieved $85 \%$ of cervical image classification accuracy. Kim and Huang (2013) detected and segmented the cancer region in high resolution cervical images only, which affects the performance of the cancer segmentation system. The authors used data driven approach to detect the cancer regions in cervical images. The authors achieved $75 \%$ of sensitivity, $76 \%$ of specificity and $75.5 \%$ of cancer segmentation accuracy.

Mouelhi et al., (2013) applied various feature extraction methods on cervical images to detect the tumor region. The authors extracted texture features, histogram features which were obtained using orientation method and color features from cervical images. These features were formatted using geometric contour model and further classified using binary classifier. Bergmeir et al., (2012) used quasi supervised learning classification approach to classify the source cervical image into either normal or abnormal. The authors extracted GLCM features and color histogram features from the cervical image and these feature set were trained and classified using quasi classification approach. The authors achieved $88 \%$ of cancer region detection positive rate and $19 \%$ cancer region negative rate.

\section{Materials and Methods}

\section{Materials}

Guanacaste dataset was created in 1997 by National Cancer Institute (NCI) (NCCC, 2010) in Guanacaste project and updated in every year (http://www.nccconline.org/index.php/cervicaltumor). This dataset contains cervical images in various categories as patient with cancer, patient without any abnormal lesions, but later developed cancer at follow-up and healthy persons, who never have the abnormal lesions in cervix area. In this paper, 50 cervical images are obtained from this open access dataset. These 50 cervical images are categorized into benign cases (35 images) and malignant cases (15 images). The benign case is the biopsy case which may or may not be developed as cancerous cells and malignant case is the cancerous case. This dataset also contains ground truth or gold standard images which are obtained and verified by expert radiologist.

\section{Methods}

The proposed cervical cancer detection and segmentation methodology is depicted in Figure 2. It consists of image registration, feature extraction, classification and segmentation. Image registration is applied on the source cervical image with respect to reference cervical image. The features are extracted from registered cervical image in order to differentiate the normal image from abnormal image. Then, these extracted features are trained and classified (cancerous or non-cancerous cervical image) using ANFIS classifier.

\section{Proposed Methodology \\ Image Registration}

It is used to register the inclined cervical image with respect to center aligned cervical image. In this paper, FFT transform is used to register the cervical image in aligned format. The source and reference RGB cervical image is converted into Luminance $(\mathrm{Y})$ and Chrominance $(\mathrm{CbCr})$ format. The FFT transform is applied on luminance of the inclined cervical image and reference cervical image in order to obtain its transformed multi resolution coefficients. The multi resolution coefficients in inclined cervical image are up sampled by up sampling factor 2. Now, these two coefficients matrix are convolved in linear manner. Inverse Fast Fourier Transform (IFFT) is now applied on the convolved coefficients matrix in order to obtain the registered cervical image. Figure 3(a) shows the reference cervical image and Figure 3(b) shows the inclined source cervical image. This inclined source cervical image is registered with respect to reference cervical image. The registered cervical image is shown in Figure 3(c).

\section{Feature Extraction GLCM features}

These energy oriented feature set clearly differentiates the normal patterns from abnormal patterns in cervical images. The GLCM matrix is constructed at the orientation of 450 in registered cervical image in order to obtain high level of classification accuracy. From the GLCM matrix, the following energy related features are extracted.

$$
\begin{aligned}
& \text { Contrast }=\sum\left(|\mathrm{i}-\mathrm{j}|^{2 \times p}(\mathrm{i}, \mathrm{j})\right) \\
& \text { Energy }=\sum \mathrm{p}(\mathrm{i}, \mathrm{j})^{2} \\
& \text { Entropy }=-\sum \mathrm{p}(\mathrm{i}, \mathrm{j})\left[\log _{2} \mathrm{p}(\mathrm{i}, \mathrm{j})\right] \\
& \text { Correlation }=\sum\left(\mathrm{i}-\mu_{\mathrm{i}}\left(\mathrm{j}-\mu_{\mathrm{j}}\right) \mathrm{p}(\mathrm{i}, \mathrm{j}) /\left(\left[\sigma_{\mathrm{i}} \cdot \sigma_{\mathrm{j}}\right]\right)\right.
\end{aligned}
$$

Where, ' $i$ ' and ' $j$ ' relates the row and column of the GLCM matrix and $p(i, j)$ represents the corresponding values in GLCM matrix.' $\sigma$ ' depicts the variance of the GLCM matrix.

Table 1 shows the extracted GLCM features for both cancerous cervical image (Malignant case) and non-cancerous cervical image (Benign case). The cancerous cervical image produces contrast about 
$1.066 * 10^{4} \pm 2.1$, Correlation about $-0.0424 \pm 1.72$, Energy about $1.654 * 10^{-5} \pm 1.62$ and Homogeneity about $0.0376 \pm 0.2$. The non-cancerous cervical image generates contrast about $1.045^{*} 10^{4} \pm 2.8$, Correlation about --0.0328 \pm 1.45 , Energy about $1.62 * 10^{-5} \pm 1.98$ and Homogeneity about $0.0344 \pm 0.32$.

\section{Grey level features}

The texture patterns from cervical images are analyzed using grey level features. The abnormal regions in cervical images have more number of cross texture patterns than the normal regions in cervical images. This grey level features correlates the relationship between the pixels in sub window and pixels in registered image. The sub window $(\mathrm{SW})$ is constructed as a $3 * 3$ matrix which is the small region of the registered image $\mathrm{I}(\mathrm{x}, \mathrm{y})$. This sub window is placed at the beginning of the cervical preprocessed image for computing the grey level feature set. Then, this window is moved from one position from current pixel. This procedure is followed at the end of final pixel in cervical preprocessed image. The following equations are used to extract the grey level features from the registered cervical image.

$$
\begin{aligned}
& P_{1}(x, y)=I(x, y)-\min \{S W\} \\
& P_{2}(x, y)=\max \{S W\}-I(x, y) \\
& P_{3}(x, y)=\operatorname{abs}(I(x, y))-\operatorname{mean}\{S W\} \\
& P_{4}(x, y)=\operatorname{std}\{S W\} \\
& P_{5}(x, y)=I(x, y)
\end{aligned}
$$

Where as, $\mathrm{SW}$ is the $3^{*} 3 \mathrm{sub}$ window in registered image and $\mathrm{I}(\mathrm{x}, \mathrm{y})$ is the registered cervical image. This grey level features produces five feature set image as shown in Figure 4.

\section{Trinary features}

The adjacent pixel difference variations are extracted from the pre-processed cervical image using the features called as trinary features. Trinary features which generates three coded values for each pixel in the fused cervical image as $+1,-1$ and 0 . The trinary feature value is described by the following equation as,

$$
\widehat{f_{1}}\left(x, g_{c^{\prime}} t\right)=\left|\begin{array}{l}
+1, x \geq g_{c}+t \\
0,\left|x-g_{c}\right|<t \\
-1, x \geq g_{c}-t
\end{array}\right| x=g_{p}
$$

Where, ' $g c$ ' is the center pixel and ' $g \mathrm{p}$ ' is the surrounding pixel. ' $t$ ' represents the variable which ranges from 0 to 255. In this paper, ' $t$ ' value is set to 4 . This threshold value is set after several iterations on preprocessed cervical image. The extracted trinary feature images are shown in Figure 5(a) and Figure 5(b).

\section{ANFIS Classification}

Classification is used to classify the source cervical image into either cancerous cervical image or non-cancerous cervical image. In this paper, ANFIS classifier is adopted for the cervical image classifications. This classification module has two phases as learning and classification. In learning phase of the ANFIS classification, the features from cancerous and non- cancerous cervical images are trained in order to produce the trained patterns. In classification phase of the ANFIS classifier, the features which are extracted from source test cervical image are classified with respect to trained patterns. The classification phase of this classifier produces either low or high binary value. The low binary value corresponds to the non-cancerous cervical image and high binary value corresponds to the cancerous cervical image.

The internal architecture of the proposed ANFIS architecture is depicted in Figure 6. This architecture receives two feature inputs $\mathrm{X}$ and $\mathrm{Y}$, respectively. In training phase, the features from cancerous cervical image and non-cervical cancerous image is represented by $\mu \mathrm{A}(\mathrm{x})$ and $\mu \mathrm{B}(\mathrm{y})$, respectively. The nodes in layer1 of this ANFIS architecture are depicted as A1, A2, B1 and $\mathrm{B} 2$. The weight of nodes in each layer is updated using the following equation as,

$$
\mathrm{Wi}=\mu \mathrm{A}(\mathrm{x}) * \mu \mathrm{B}(\mathrm{y}) ; \mathrm{i}=1,2 \text {. }
$$

The functional factor of the nodes in layer is computed as,

$\mathrm{f}=(\mathrm{W} 1 * \mathrm{X}+\mathrm{W} 2 * \mathrm{Y}) /(\mathrm{W} 1+\mathrm{W} 2)$

The inverse weighting function can be expressed as,

$$
\text { w_inv_i=w1/(w1+w2), } \mathrm{i}=1,2 \text {. }
$$

The final response of the ANFIS architecture is given in the following expression using its inverse weighting functions and functional factor.

$$
\mathrm{f}=\mathrm{w} \_ \text {inv_1 } 1 * \mathrm{f} 1+\mathrm{w} \_ \text {inv_2 } 2 * \mathrm{f} 2
$$

\section{Cancer region segmentation}

Segmentation is an important process in order to detect the abnormal patterns from the normal patterns in classified cervical images. The unsharp contrast enhancement filter (Mithilesh Kumar et al., 2016) is applied on the classified abnormal cervical image in order to smooth the edge regions in image. Then, the pixel value less than 80 are choosing to form the threshold image. This threshold value is set after many several trail and test. Now, morphological opening function with disc shaped structuring element 2 is applied over this threshold image to smoothening the outer edges in threshold image.

The morphological opening function on cervical image (I) with structuring element $(\mathrm{m})$ is given in the following equation.

$$
I \circ h=(I \ominus m) \oplus m
$$

The morphological closing function on cervical image (I) with structuring element (m) is given in the following equation.

$$
I \bullet h=(I \oplus m) \ominus m
$$

The threshold image is shown in Figure 7(a). Then, 'Sobel' edge detectors are applied on this threshold image, which produces the fine edge regions in the cervical image 
as abnormal edges. The abnormal edge pixels are shown in Figure 7(b). Finally, the overlay abnormal edge pixels in source cervical image are shown in Figure 7(c).

Figure 8(a) shows the source cervical images which are obtained from open access dataset. Figure 8(b) shows the cancer region segmentation result by proposed method and Figure 8(c) shows the ground truth images which are obtained from expert radiologist.

\section{Results}

The proposed cervical cancer detection and segmentation methodology is evaluated in MATLAB R2014b simulating tool (programming language). The proposed algorithm is executed on Intel Dual core i3 processor with $4 \mathrm{~GB}$ internal RAM as memory. In this paper, ANFIS classifier based morphological operations are used to detect and segment the cancer region in cervical images. The morphological property of the segmented cancer region is analyzed in terms of perimeter, area, width and height using the following expressions.

$$
\begin{aligned}
& \text { Perimeter }=2 * \pi * r \\
& \text { Area }=\pi * r^{2} \\
& \text { Width }=\text { Height }=2 * r
\end{aligned}
$$

Where as, radius of the segmented cancer region in cervical image is noted as ' $r$ '. Width and height of the segmented cancer region is measured in horizontal and vertical axis through the center point of the segmented cancer region. Table 2 shows the morphological analysis of the segmented cancer region using the equations (17)(19). It gives the morphological values for both benign

Table 1. Extracted GLCM Features for Cancerous and Non-Cancerous Cervical Image

\begin{tabular}{lcc}
\hline Parameters & $\begin{array}{c}\text { Cancerous } \\
\text { cervical image } \\
\text { (Malignant case) }\end{array}$ & $\begin{array}{c}\text { Non-cancerous } \\
\text { cervical image } \\
\text { (Benign case) }\end{array}$ \\
\hline Contrast & $1.066^{*} 10^{4} \pm 2.1$ & $1.045^{*} 10^{4} \pm 2.8$ \\
Correlation & $-0.0424 \pm 1.72$ & $-0.0328 \pm 1.45$ \\
Energy & $1.654^{*} 10^{-5} \pm 1.62$ & $1.62 * 10^{-5} \pm 1.98$ \\
Homogeneity & $0.0376 \pm 0.2$ & $0.0344 \pm 0.32$ \\
\hline
\end{tabular}

\begin{tabular}{|c|c|c|}
\hline \multirow[t]{2}{*}{ Parameters } & \multicolumn{2}{|c|}{ Experimental Results } \\
\hline & $\begin{array}{l}\text { For benign } \\
\text { cancer region }\end{array}$ & $\begin{array}{l}\text { For malignant } \\
\text { cancer region }\end{array}$ \\
\hline Perimeter $(\mu \mathrm{m})$ & $325.7 \pm 2.75$ & $845.2 \pm 1.72$ \\
\hline $\operatorname{Area}\left(\mu \mathrm{m}^{2}\right)$ & $2563 \pm 2.78$ & $4192 \pm 3.28$ \\
\hline Width $(\mu \mathrm{m})$ & $36.7 \pm 3.28$ & $112 \pm 2.61$ \\
\hline Height $(\mu \mathrm{m})$ & $39.6 \pm 1.93$ & $128 \pm 2.58$ \\
\hline
\end{tabular}

Table 2. Morphological Analysis of the Segmented Cancer Region

Table 3. Performance Analysis of Proposed System

\begin{tabular}{lc}
\hline Parameters & Experimentation Results (\%) \\
\hline Sensitivity & 97.42 \\
Specificity & 99.36 \\
Accuracy & 99.36 \\
\hline
\end{tabular}

Table 4. Performance Comparisons

\begin{tabular}{lccc}
\hline Methodologies & $\begin{array}{c}\text { Sensitivity } \\
(\%)\end{array}$ & $\begin{array}{c}\text { Specificity } \\
(\%)\end{array}$ & $\begin{array}{c}\text { Accuracy } \\
(\%)\end{array}$ \\
\hline Proposed work & 97.42 & 99.36 & 99.36 \\
Dezhao Song et al. (2015) & 83.21 & 94.79 & - \\
Kim and Huang (2013) & 75 & 76 & 75.5 \\
\hline
\end{tabular}

and malignant cases.

The proposed cervical cancer detection system is applied over the set of cervical images which are available in publicly accessible dataset. In this paper, 50 cervical images (non-cancerous images: 35 and

(a)

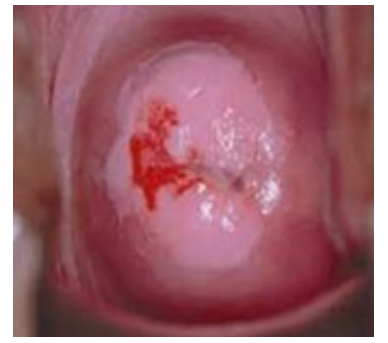

Figure 1. Cervical Images (a) Normal case (b) Abnormal case

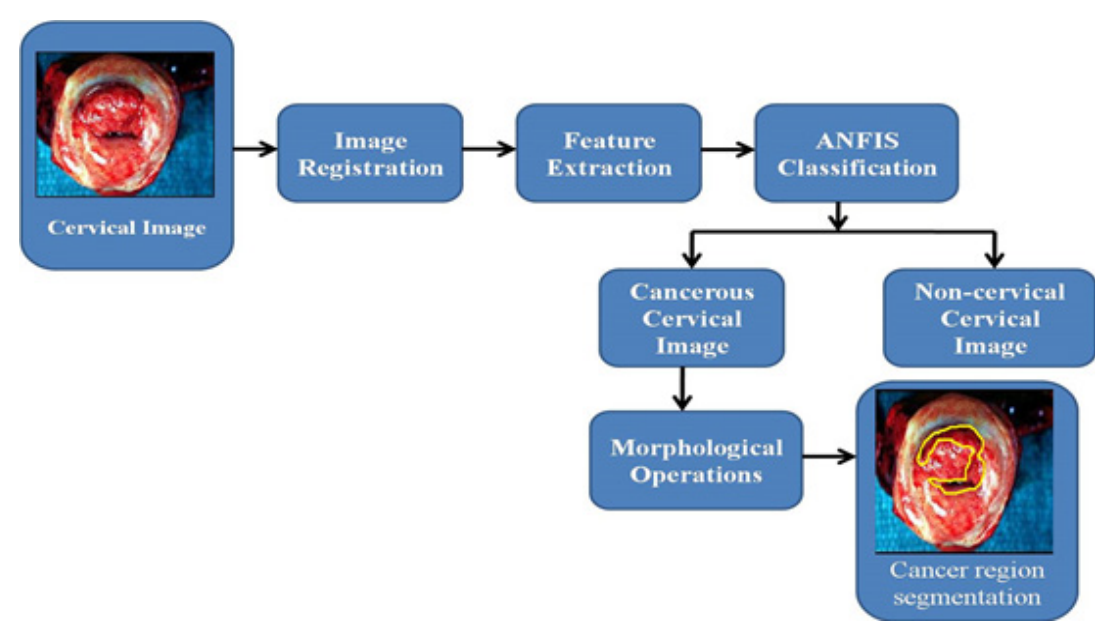

Figure 2. Proposed Flow for Cervical Cancer Detection and Segmentation 
(a)

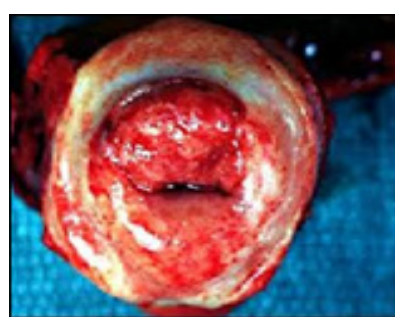

(b)

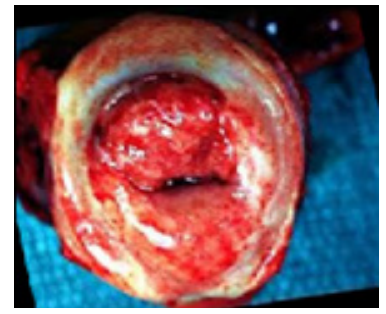

(c)

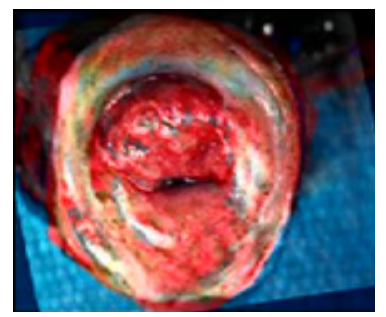

Figure 3. (a) Reference cervical image (b) Source cervical image (c) Registered cervical image

(a)

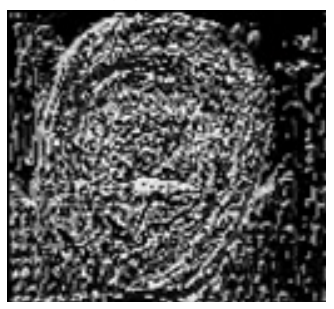

(b)

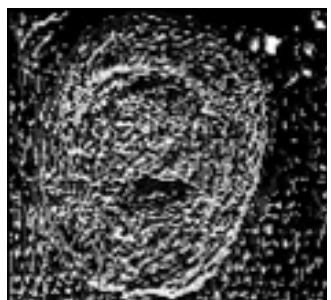

Figure 5. (a) Trinary feature image1 (b) Trinary feature image 2

images. Hence, the proposed system achieves $100 \%$ of classification accuracy for cancerous cervical images. This proposed methodology for cervical images classification achieves $98.57 \%$ of overall classification accuracy.

The performance of the proposed cervical cancer detection and segmentation system is analyzed in terms of sensitivity, specificity and accuracy as stated below.

$$
\begin{aligned}
& \text { Sensitivity }=\frac{T R P O}{T R P O+F A N E} \\
& \text { Specificity }=\frac{T R N E}{T R N E+F A P O} \\
& \text { Accuracy }=\frac{T R P O+T R N E}{T R P O+F A P O+T R N E+F A N E}
\end{aligned}
$$

Where, TRPO is True Positive, which is the number of correctly detected cancer pixels as cancer pixels, TRNE is True Negative, which is the number of correctly detected non-cancer pixels as non-cancer pixels, FAPO is False Positive, which is the number of wrongly detected cancer pixels as non-cancer pixels and FANE is False Negative,

(a)

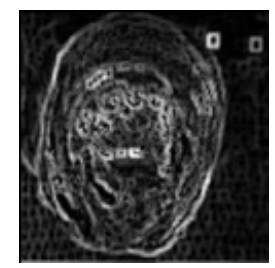

(d)

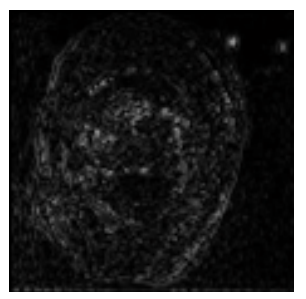

(b)

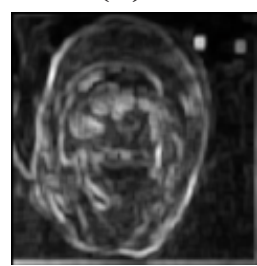

(e)

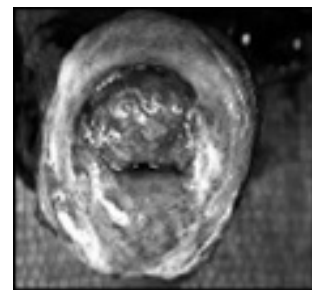

Figure 4. Extracted Grey Level Features (a) P1(x,y) (b) P2 (x,y) (c) P3(x,y) (d) P4(x,y) (e) P5(x,y)

(c)

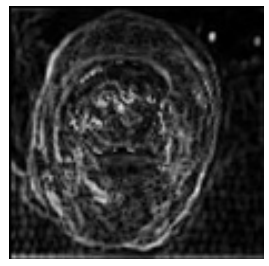

(b)
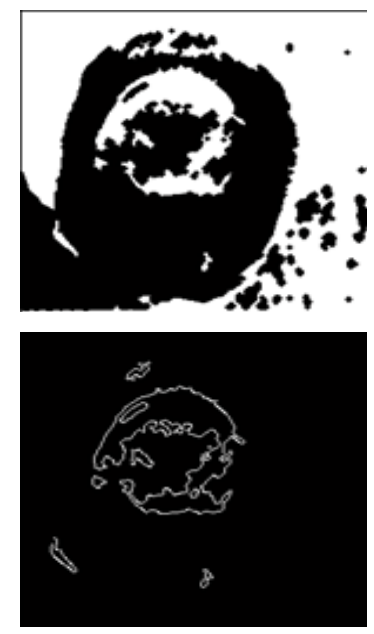

(c)

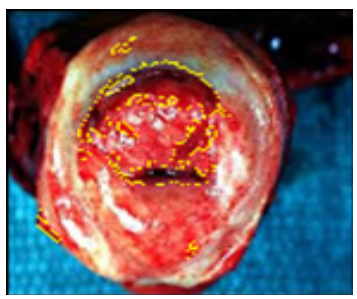

Figure 7. (a) Thresholded image (b) Abnormal edges detected image (c) Cancer region segmented image 

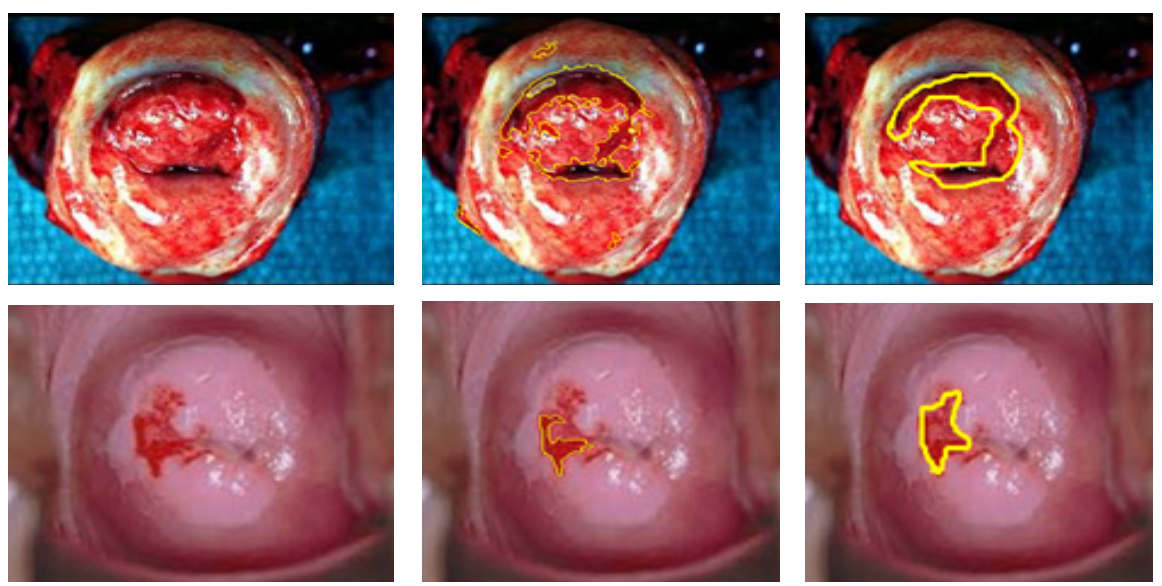

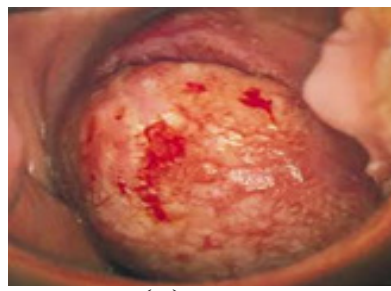

(a)

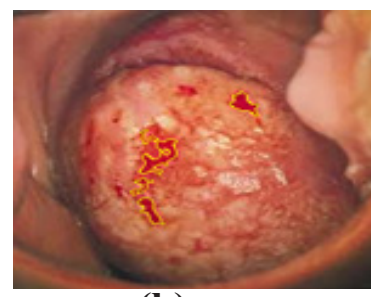

(b)

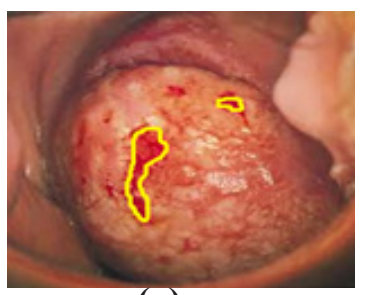

(c)

Figure 8. (a) Source cervical images (Available at: http://www.nccc-online.org/index.php/cervicaltumor) (b) Cancer region segmentation by proposed method (c) Ground truth images

which is the number of wrongly detected non-cancer pixels as cancer pixels.

\section{Discussion}

Table 3 shows the performance analysis of proposed cervical cancer detection and segmentation system achieves $97.42 \%$ of sensitivity, $99.36 \%$ of specificity and $99.36 \%$ of segmentation accuracy.

Table 3 shows the performance of the proposed cervical cancer detection and segmentation system with conventional methodologies as Dezhao et al., (2015) and Kim and Huang (2013). Dezhao et al., (2015) segmented the inner region of the cancer area in cervical image which reduced the cancer region segmentation accuracy with respect to ground truth images. Kim and Huang (2013) detected and segmented the cancer region in high resolution cervical images only, which affects the performance of the cancer segmentation system. The proposed system achieves $14.58 \%$ of improvements in sensitivity and $4.5 \%$ of improvements in specificity with conventional method Dezhao et al., (2015). The proposed system achieves $23.01 \%$ of improvements in sensitivity and $23.51 \%$ of improvements in specificity and $24.01 \%$ of improvements in accuracy with conventional method Kim and Huang (2013). From Table 4, the proposed methodology stated in this paper for cervical cancer detection and segmentation achieves better results when compared with conventional methods.

In this paper, MATLAB R2014b is used to simulate the proposed cervical cancer detection and segmentation system with 4GB RAM and Intel core i3 processor as hardware specifications.

The automatic detection and segmentation of cancer region in cervical images plays an important role in cervical cancer diagnosis. In this paper, image registration based cervical cancer detection and segmentation methodology is proposed using ANFIS classifier. The proposed system achieves $97.14 \%$ and $100 \%$ of classification accuracy for non-cancerous and cancerous cervical images, respectively. This proposed methodology for cervical images classification achieves $98.57 \%$ of overall classification accuracy. The performance analysis of proposed cervical cancer detection and segmentation system achieves $97.42 \%$ sensitivity, $99.36 \%$ specificity and $99.36 \%$ segmentation accuracy. Simulations on different aspects prove that the proposed methodology stated in this paper achieves high performance for clinical practice. In future, the temporal and spatial relationship between each pixel is considered for improving the performance of the cervical cancer detection and diagnosis process.

\section{References}

American Cancer Society (ACS) (2011). 'What is cervical tumor?,'. Available at: http://www.tumor.org/Tumor/ CervicalTumor/Detailed-Guide/cervical-tumor-what-iscervical-tumor.

Bergmeir C, Silvente MG, Benitez JM (2012). Segmentation of cervical cell nuclei in high-resolution microscopic images: a new algorithm and a web-based software framework. Comput Methods Programs Biomed, 107, 497-512.

Bhattacharjee S, Mukherjee J, Nag S, Maitra IK, Bandyopadhyay SK (2014). Review on histopathological slide analysis using digital microscopy. Int J Adv Sci Technol, 62, 65-96.

Chen YF, Huang PC, Lin KC, et al (2014). Semi-automatic segmentation and classification of pap smear cells. IEEE IEEE J Biomed Health Inform, 18.

Cronje HS (2005). Screening for cervical tumor in the developing world. Best Pract Res Clin Obstet Gynaecol, 19, 517-29.

Dezhao S, Edward K, Xiaolei H, et al (2015). Multimodal entity 
coreference for cervical dysplasia diagnosis. IEEE Trans Med Imaging, 34, 1.

Http://www.cse.lehigh.edu/ idealab/cervitor/ downloads.html.

Kim E, Huang X (2013). A data driven approach to cervigram image analysis and classification. Color Medical Image Analysis, ser. Lecture Notes in Comput. Vis. Biomechan., M. E. Celebi and G. Schaefer, Eds. Amsterdam, The Netherlands: Springer, 6, pp 1-13.

Kumar R, Srivastava R (2014). Some observations on the performance of segmentation algorithms for microscopic biopsy images. Proceedings of the International Conference on Modeling and Simulation of Diffusive Processes and Applications, pp 16-22.

Kashyap D, Abhishek S, Jatin S, et al (2016), Cervical cancer detection and classification using Independent Level sets and multi SVMs. 39th International Conference on Telecommunications and Signal Processing (TSP), Vienna, pp 523-8.

Mithilesh K, Ashima R (2016). Image enhancement using contrast limited adaptive histogram equalization and wiener filter. Int J Engg Comput Sci, 5, 16977-9.

Mouelhi A, Sayadi M, Fnaiech F, Mrad K, Romdhane KB (2013). Automatic image segmentation of nuclear stained breast tissue sections using color active contour model and an improved watershed method. Biomed Signal Process Control, 8, 421-36.

Miri MR, Moodi M, Sharif-Zadeh G-R, et al (2018), Cognitive predictors of cervical cancer screening's stages of change among sample of Iranian women health volunteers: A path analysis. PLoS One, 13, 245-76.

NCCC (2010). Cervical tumor, Available at: http://www.nccconline.org/index.php/cervicaltumor.

Parkin DM, Bray F, Ferlay J, Pisani P (2005). Global tumor statistics. Ca-Tumor J Clin, 55, 74.

Rama Praba PS, Ranganathan H (2013). Comparing different classifiers for automatic lesion detection in cervix based on colour histogram. J Comput Appl, 6, 1 .

Soumya MK, Sneha K, Arunvinodh C (2016). Cervical cancer detection and classification using texture analysis. Biomed Pharmacol J, 9, 123-34.

Sachan PL, Singh M, Patel ML, Sachan R (2018). A study on cervical cancer screening using pap smear test and clinical correlation. Asia Pac J Oncol Nurs, 5, 337- 41.

\section{(c) (i) (8)}

This work is licensed under a Creative Commons AttributionNon Commercial 4.0 International License. 